\title{
Equivalent dose in 2 Gy (EQD2) to pelvic lymph nodes using volume based prescription for three brachytherapy applicators - a dosimetric retrospective analysis
}

\author{
Revathy T, Nishant Vidyasagar ${ }^{\circledR}$, Janaki Manur Gururajachar, Arul Ponni Thiruraj, Kirthi Koushik AS, \\ Ram Charith Alva, Mohan Kumar S \\ Department of Radiation Oncology, Ramaiah Medical College, Bengaluru, India
}

\begin{abstract}
Background: Pelvic lymph node (PLN) metastasis has been included in the FIGO staging, so there is a need to determine the dose contribution from brachytherapy to ascertain the total delivered dose to the pelvic lymph nodes in cervical cancer. The aim of the study was to calculate the equivalent dose in $2 \mathrm{~Gy}$ (EQD2) of the pelvic lymph nodes (PLNs) based on volume prescription using three applicators.

Materials and methods: Forty-one patients who had undergone external beam radiotherapy followed by brachytherapy using tandem ovoids (TO), tandem ring (TR) and TO + free hand interstitial needles (TO + FH) applicators were taken for this study. 26 Gy in 4 fractions was prescribed to HRCTV. The external iliac node (ELN), internal iliac node (ILN) and obturator (OBT) were contoured and the median EQD2 of the lymph nodes was calculated.

Results: The median bilateral EQD2 values of ELN were 1.55 Gy (TR), 1.75 Gy (TO), 1.9 Gy (TO + FH), of ILN these were 2.57 Gy (TR), 3.27 Gy (TO), 3.04 Gy (TO + FH), and of OBT these were 3.69 Gy (TR), $4.46 \mathrm{~Gy}$ (TO), $4.69 \mathrm{~Gy}$ (TO + FH), respectively. The total median EQD2 values of TR, TO and TO + FH were 52.71 Gy, 53.03 Gy, and 53.88-62.73 Gy, respectively.

Conclusion: Our study calculated the median EQD2 to the pelvic lymph nodes using three types of applicators in brachytherapy. This could serve as reference to decide on the EBRT boost dose while treating patients with enlarged pelvic lymph nodes.

Key words: pelvic lymph nodes; HDR; brachytherapy; EQD2; Co-60; applicators

Rep Pract Oncol Radiother 2021;26(3):408-415
\end{abstract}

\section{Introduction}

Cervical cancer is one of the most common malignancies in developing countries. External beam radiation therapy (EBRT) to a dose of 45 to 50.4 Gy in 25 to 28 fractions with concurrent chemotherapy followed by brachytherapy (BT) to a dose of 6 to 9 Gy per fraction in 2 to 4 fractions is the standard care for locally advanced cervical cancer. Traditionally, carcinoma of the uterine cervix was staged clinically, but now radiological methods are incorporated in assigning stage to the disease due to their impact on treatment [1]. Moreover, metastasis to the pelvic lymph nodes (PLNs) is one of the most important prognostic factors which has also been included in the FIGO staging [2]. Some retrospective studies have shown only minimal or no benefit in terms of local control and overall survival with dose escalation using EBRT [3]. The dose contribution to the pelvic nodes from brachytherapy is gen-

Address for correspondence: Dr Nishant Vidyasagar, Senior Resident, Department of Radiation Oncology, Ramaiah Medical College and Hospital, Bengaluru, India 560054, tel: +91 99513071273; e-mail: nishvidsar8391@gmail.com

This article is available in open access under Creative Common Attribution-Non-Commercial-No Derivatives 4.0 International (CC BY-NC-ND 4.0) license, allowing to download articles and share them with others as long as they credit the authors and the publisher, but without permission to change them in any way or use them commercially 
erally neither documented nor reported because it constitutes a very small part of the prescribed dose. Furthermore, there are no standardized guidelines that determine the total dose required to achieve a complete tumor response in the nodal regions. Considering its prognostic significance and the absence of consensus, there is a need to determine the dose contribution from brachytherapy to ascertain the total delivered dose to the pelvic lymph nodes in cervical cancer.

Intracavitary brachytherapy (ICBT) involves placement of central tandem in the uterine cavity along with the ovoids or ring in the vaginal fornices. But in cases where there is minimal involvement of the parametrium beyond the medial half of the lateral pelvic wall and cervical os could be sounded, intracavitary brachytherapy with free hand interstitial needles can be used. This type of application is regarded as intracavitary + interstitial brachytherapy [4].

BT has undergone a paradigm shift from X-ray based planning to image guided planning using computed tomography (CT) or magnetic resonance imaging (MRI). With the advent of image guided brachytherapy, it has become easier to delineate the target and organs at risk which has, in turn, helped in improving local control and minimizing long term side effects [5]. Most centres across the globe use the conventional point based prescription and in literature there are few studies that estimate the dose contribution to PLNs in brachytherapy using point based prescription.

The aim of the study was to calculate the equivalent dose in 2 Gy (EQD2) to the external iliac lymph node (ELN), the internal iliac lymph node (ILN) and the obturator (OBT) group of lymph nodes using CT based high dose rate (HDR) BT with three different applicators: tandem and ovoid (TO), tandem and ring (TR), tandem and ovoid with free hand interstitial needles (TO $+\mathrm{FH})$ using volume based prescription.

\section{Materials and methods}

With institutional ethical board clearance, 41 cervical cancer patients from stage IB2 to IIICr1 who underwent BT from January 2018 to January 2019 treated at a tertiary care hospital (Ramaiah Medical College Hospital) were included in this retrospective study. All patients underwent biopsy from the growth, complete blood count, renal function tests, liver function tests, echocardiogram and contrast enhanced CT scan of the abdomen and pelvis.

\section{External beam radiotherapy (EBRT)}

Patients were treated with EBRT to a dose of $45 \mathrm{~Gy}$ in 25 fractions using the three dimensional conformal radiation therapy (3DCRT) technique or intensity modulated radiation therapy (IMRT) on 6MV Elekta Agility Linear accelerator with concurrent weekly cisplatin chemotherapy. Bladder, rectum, bowel and bilateral femoral heads were contoured as organs at risk (OARs). The planning target volume included the entire uterus, bilateral parametria and upper half or entire vagina depending on the extent of the disease along with bilateral common iliac, external iliac, internal iliac, obturator and presacral group of lymph nodes that were included up to the S2-S3 junction. The OARs dose constraints were V45 less than $50 \%$ for the bladder, rectum and less than $5 \%$ for femur, respectively. $195 \mathrm{cc}$ volume of the bowel bag should receive less than 45 Gy. The PTV should receive more than 95\% of the prescription dose. Those patients who had an enlarged node of more than one centimetre received a boost of 5.4 to 9 Gy/3-5 fractions depending on the dose to OARs and technique of radiation.

\section{Brachytherapy details}

Patients received brachytherapy in four fractions to a dose of 6.5 Gy per fraction around two weeks post completion of EBRT.

Patients were examined under anaesthesia to assess the response to EBRT and the type of ICBT application was decided based on the examination findings. In patients with central disease and retained cervix, TR ( $\operatorname{arm}$ A) was used. Patients who had residual disease confined to medial $1 / 3^{\text {rd }}$ of the parametrium underwent TO ( $\operatorname{arm} B)$. Patients with an unilateral residual parametrium disease beyond medial $1 / 3^{\text {rd }}$ underwent TO $+\mathrm{FH}(\operatorname{arm} \mathrm{C})$.

CT scan was done after the procedure with $3 \mathrm{~mm}$ axial slices (Asteion VP). The images were loaded into the HDR plus 3.0.8 treatment planning system (TPS) based on Task Group (TG-43) algorithm [6]. The bladder, rectum, sigmoid and high risk clinical target volume (HRCTV) were contoured based on Viswanathan et al. CT based contouring guidelines [7]. Applicators were digitized and surface control points were created based on HRCTV. Volume based planning was used and manual optimisa- 
tion was done to minimize the OAR doses and to achieve the dose intended to D90 HRCTV. Bebig Multisource Cobalt based HDR unit (Eckert and Ziegler, Germany) was used to execute the treatment on two consecutive days with a six-hour gap between two fractions.

\section{Dosimetric analysis}

In addition to the targets and OARs, the pelvic lymph nodes were contoured by a radiation oncologist according to the consensus guidelines formulated by Chua et al. [8]. The ELN, ILN, OBT group of lymph nodes were contoured for each patient bilaterally. The ELN group was contoured cranially from the L5/S1 junction or two $\mathrm{cm}$ above the top of the applicator, whichever is inferior, caudally up to the superior femoral head, a seven mm margin around the vessels, was given, except anteriorly where a $10 \mathrm{~mm}$ margin was used. The ILN group was contoured cranially from the L5/S1 junction or two $\mathrm{cm}$ above the top of the applicator, whichever is inferior, caudally up to the termination of the internal iliac vessels, a seven $\mathrm{mm}$ isotropic margin was given around the vessels. The OBT group was contoured from the commencement of the gap between the ELN and ILN regions and connecting the two regions with a seven $\mathrm{mm}$ margin around vessels up to the obturator foramen. All the lymph node contours were trimmed from the OARs. Figures 1-3 show the axial sections of the contours of
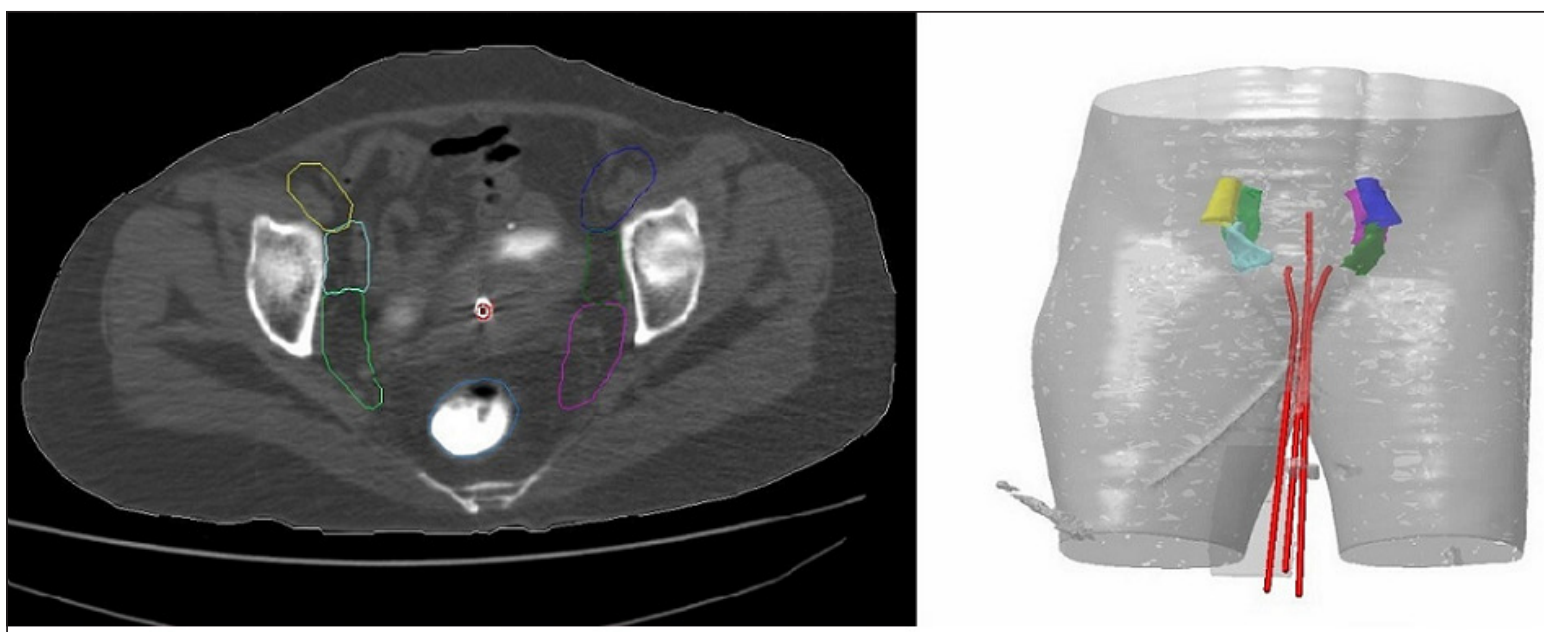

Keys: Right external Left external

Right obturator

Left obturator

Right internal Left internal

Figure 1. Axial and 3D window of Tandem and ring application
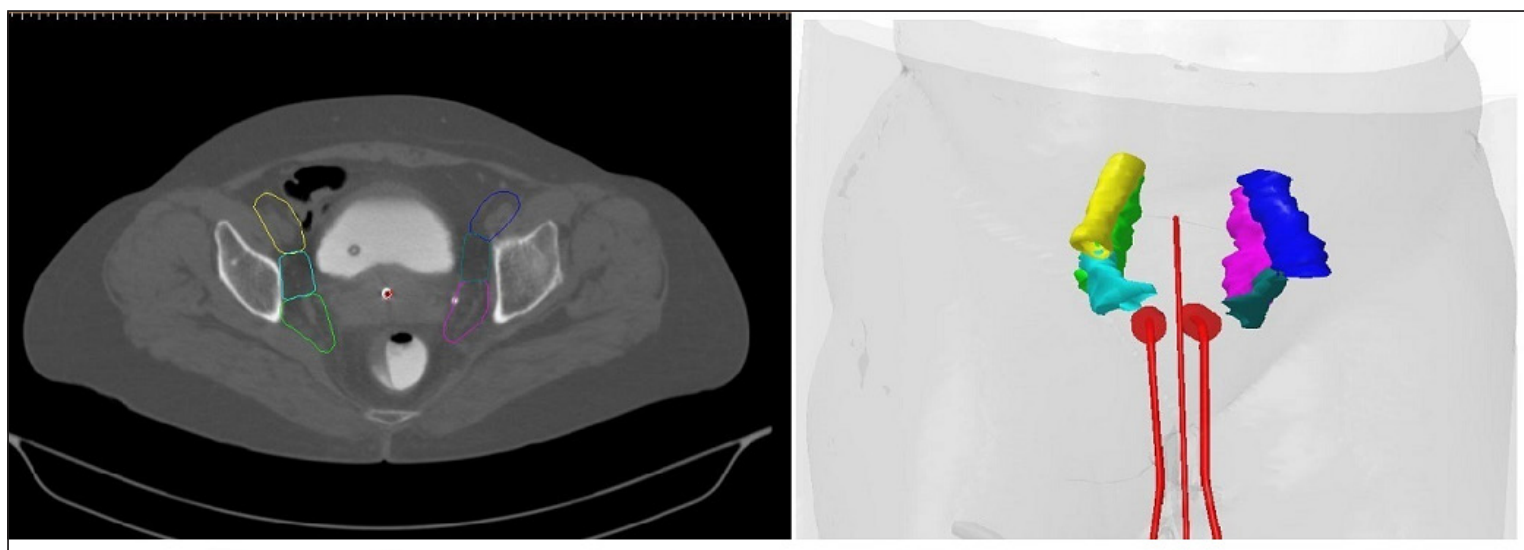

Keys: $\quad$ Right external

Left external

Right obturator

Left obturator

Right internal

Left internal

Figure 2. Axial and 3D window of Tandem and ovoid application 


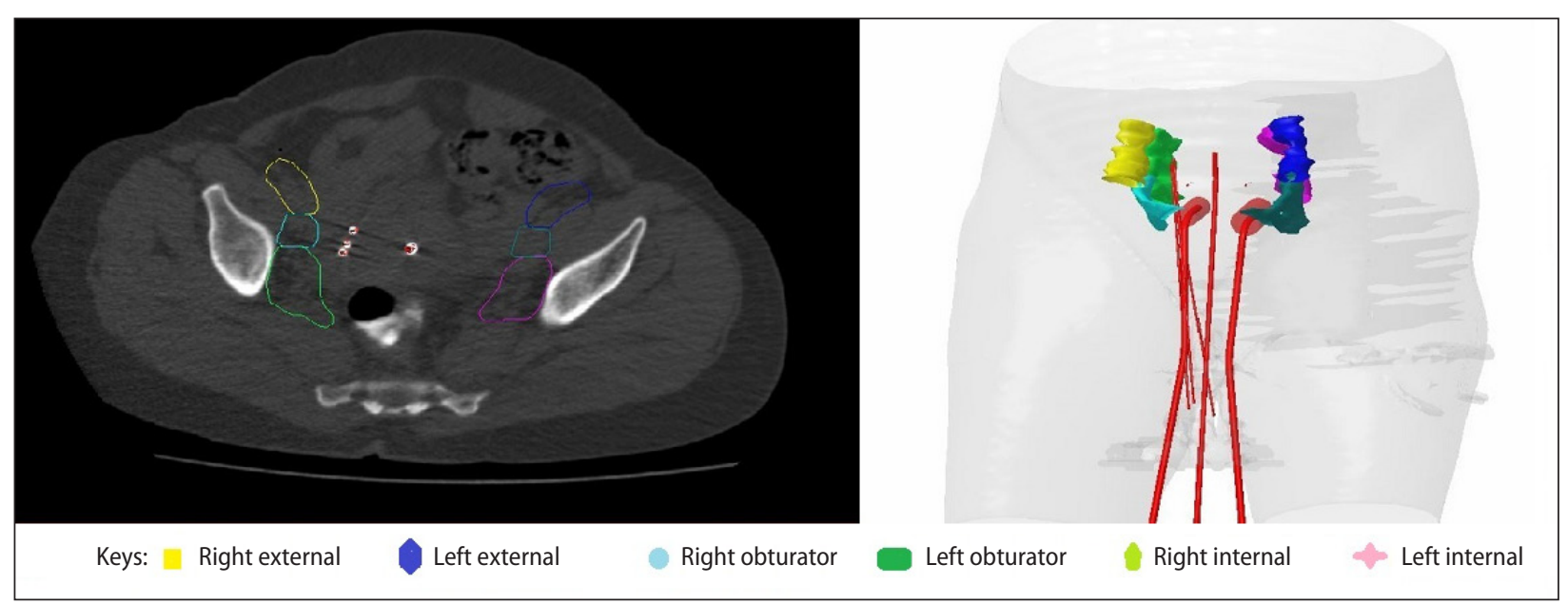

Figure 3. Axial and 3D window of Tandem ovoid with free hand interstitial needles

Table 1. Patient characteristics

\begin{tabular}{|l|c|}
\hline No. of patients & $41(100 \%)$ \\
\hline Age & \\
Median & 55 \\
Range & $35-70$ \\
\hline Stage & \\
IB & $1(2 \%)$ \\
IIA & $8(20 \%)$ \\
IIB & $21(51 \%)$ \\
IIIB & $9(22 \%)$ \\
IIICr1 & $2(5 \%)$ \\
\hline Applicators & \\
Tandem and ring & $15(37 \%)$ \\
Tandem and ovoid & $15(37 \%)$ \\
Tandem and ovoid + free hand & $11(26 \%)$ \\
\hline
\end{tabular}

the pelvic lymph node groups for three different applicators.

The mean dose to each nodal group was documented for all three arms. The cumulative median dose to each lymph node group was calculated and tabulated. The equivalent dose to 2 Gy (EQD2) was calculated using the below formula:

$$
E Q D 2=n d[(1+d / \alpha / \beta) /(1+2 / \alpha / \beta)]
$$

$\mathrm{n}-$ number of fractions; $\mathrm{d}-$ dose per fraction; $\alpha / \beta-10$ for tumor.

\section{Statistical methods}

The values were tabulated and analysed using SPSS version 18.0 software (SPSS Inc., IBM, Chicago, USA). Kruskal Wallis Test was used to compare the median EQD2 of the pelvic lymph nodes across the three arms. A Friedman test was used to compare point $\mathrm{B}$ EQD2 and median EQD2 to 2cc pelvic lymph nodes for all the applicators. A Spearman correlation coefficient test was used to study the correlation between point B EQD2 and the median EQD2 to 2cc pelvic lymph nodes. The values were considered statistically significant when the $\mathrm{p}$ value was less than 0.05 .

\section{Results}

Patient characteristics are shown in Table 1 and the median age was 55 years (35-70). Twenty-nine patients had stage IIA-IIB, nine patients had stage IIIB, two patients had stage III Cr1 and one patient had stage IB. Among 41 patients in our study, 15 patients underwent ICBT with TR ( $\operatorname{arm} A), 15$ patients underwent ICBT with TO (arm B) and 11 patients underwent with $\mathrm{TO}+\mathrm{FH}(\operatorname{arm} \mathrm{C})$ of whom nine patients had stage IIIB and two patients had stage IIICr1.

Table 2 summarizes the median EQD2 to point $\mathrm{B}$ and $2 \mathrm{cc}$ of the pelvic lymph nodes for three different applicators.

Figure 4 shows the Box-and-Whisker plot of the distribution of EQD2 to 2cc pelvic lymph nodes and point $\mathrm{B} E Q D 2$ in the three arms. There is no significant difference between the arms in terms of median EQD2 to 2cc of bilateral pelvic lymph nodes [EXT $(\mathrm{p}=0.144)$; INT $(\mathrm{p}=0.747)$; OBT $(\mathrm{p}=0.608)]$.

Subsequently, the median EQD2 of the bilateral ELN, ILN and OBT nodes were compared with 
A

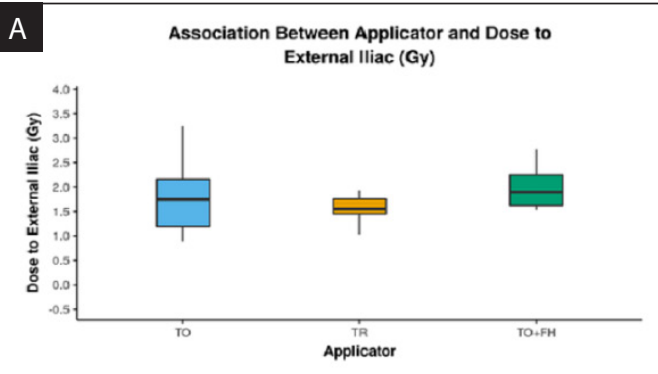

C

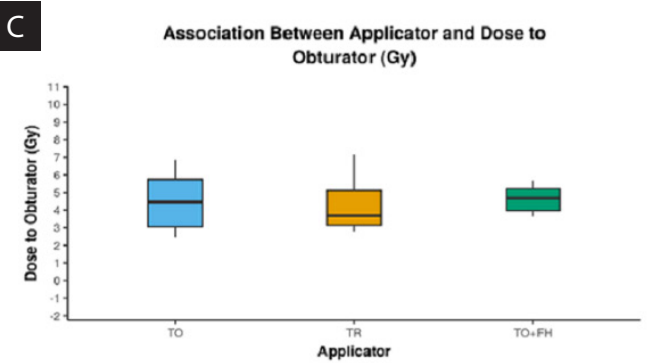

B

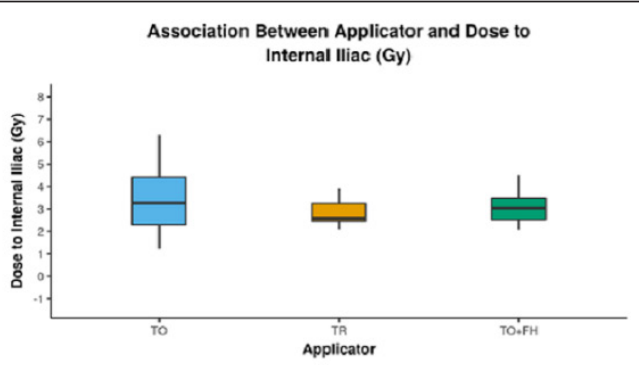

D

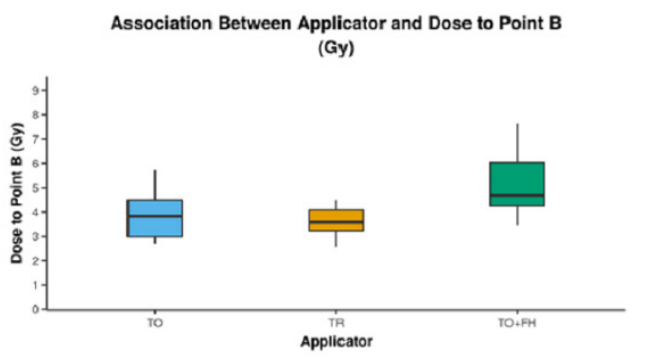

Figure 4. The Box-and-Whisker plot of equivalent dose in 2 Gy (EQD2) to pelvic lymph nodes. A. External iliac; B. Internal iliac; C. Obturator; D. Point B. The middle horizontal line represents the median EQD2 and the upper and lower bounds of the box represent the $75^{\text {th }}$ and the $25^{\text {th }}$ centile of EQD2

Table 2. Median equivalent dose in 2 Gy (EQD2) to point B and D2cc of pelvic lymph nodes for three different applicators (Krushkal Wallis Test)

\begin{tabular}{|c|c|c|c|c|c|}
\hline Parameters & $\begin{array}{l}\text { Tandem and Ring } \\
\qquad(\mathrm{n}=15) \\
\text { Median (range) }\end{array}$ & $\begin{array}{l}\text { Tandem and ovoid } \\
\qquad(\mathrm{n}=15) \\
\text { Median (range) }\end{array}$ & $\begin{array}{l}\text { Tandem Ovoid + Free Hand } \\
\qquad(\mathbf{n}=11) \\
\text { Median (range) }\end{array}$ & $\begin{array}{l}\text { Kruskal Wallis test } \\
\qquad\left(\mathrm{x}^{2}\right)\end{array}$ & p-value \\
\hline \multicolumn{6}{|c|}{ External lymph nodes (bilateral) } \\
\hline Volume [cc] & $19.75(11.5-28.25)$ & $21.45(11.15-42.4)$ & $23.75(12.8-28.85)$ & & \\
\hline EQD2 [Gy] & $1.55(1.45-1.76)$ & $1.75(1.19-2.16)$ & $1.9(1.62-2.25)$ & 3.872 & 0.144 \\
\hline \multicolumn{6}{|c|}{ Internal lymph nodes (bilateral) } \\
\hline Volume[cc] & $31.8(23.85-54.15)$ & $33.4(22.85-58.65)$ & $27.95(14.55-32.35)$ & & \\
\hline EQD2 [Gy] & $2.57(2.45-3.25)$ & $3.27(2.3-4.41)$ & $3.04(2.52-3.48)$ & 0.583 & 0.747 \\
\hline \multicolumn{6}{|c|}{ Obturator (bilateral) } \\
\hline Volume [cc] & $14.05(8.3-22.9)$ & $14.7(10.55-28.45)$ & $\begin{array}{c}16.55 \\
(12-24.35)\end{array}$ & & \\
\hline EQD2 [Gy] & $3.69(3.15-5.12)$ & $4.46(3.05-5.74)$ & $4.69(3.97-5.22)$ & 0.995 & 0.608 \\
\hline \multicolumn{6}{|l|}{ Point B } \\
\hline EQD2 [Gy] & $3.59(3.23-4.09)$ & $3.83(3-4.48)$ & $\begin{array}{c}4.69 \\
(4.25-6.03)\end{array}$ & 9.574 & 0.008 \\
\hline
\end{tabular}

median point B EQD2 for all the applicators. Point B EQD2 is significantly different from the median EQD2 to the ELN and ILN group ( $p$ value $<0.001$ ), but no such difference is seen for the OBT group ( $\mathrm{p}$ value $=0.574)$. The correlation coefficients between point $\mathrm{B}$ and the D2cc of the pelvic lymph nodes are greater than 0.8 in the TO arm, between 0.59 to 0.83 in the TR arm and less than 0.4 in the $\mathrm{TO}+\mathrm{FH}$ arm.
Table 3 depicts the total EQD2 for three applicators obtained by combining EBRT and BT EQD2.

\section{Discussion}

Locally advanced cervical cancers are treated with definitive chemoradiation followed by brachytherapy. The incidence of pelvic lymph node metastases for FIGO stage IIA is $10-45 \%, 26-62 \%$ for 
Table 3. Total equivalent dose in 2 Gy (EQD2) of three applicators obtained by combining external beam radiation therapy (EBRT) and brachytherapy (BT) EQD2

\begin{tabular}{|c|c|c|c|}
\hline Applicator & EBRT [Gy] & BT [Gy] & Total EQD2 [Gy] \\
\hline Tandem and Ring & 44.25 & 8.51 & 52.71 \\
\hline Tandem and Ovoid & 44.25 & 8.78 & 53.03 \\
\hline Tandem Ovoid + FreeHand & $44.25-53.1$ & 9.63 & $53.88-62.73$ \\
\hline
\end{tabular}

stage IIB , 39-59\% for stage IIIA, and 39-88\% for stage IIIB/IV according to some surgical series [9]. The aim is to deliver a cumulative EQD2 dose of 80-90 Gy to primary tumor using BT, after EBRT, dose of 45 to $50.4 \mathrm{~Gy}$, respectively [10]. However, dose to the pelvic nodes is less certain with BT. This study is an effort to determine the median dose to the nodal regions using three different applicators which could help in estimating EBRT boost dose. Conventionally, dose to the lymph nodes for ICBT is reported by point $B$ and the reference points of the lymphatic trapezoid. Point B dose corresponds to the dose to the obturator lymph nodes which constitute the first echelon group in cervical cancers.

Similar studies have been done by Chua et al. [8], Lee et al. [11], Lee Y et al. [12] and Matsukawa et al. [13], where they did CT based planning but prescribed to point A.

Chua et al. studied the dose to the pelvic lymph nodes using two dose fractionation schedules (5 and 6 Gy per fraction) on 40 patients. With a brachytherapy dose of 5 Gy to point $\mathrm{A}$, the mean EQD2 doses received by the EI, II and OB groups were $0.71,1.04$, and $1.27 \mathrm{~Gy}$, respectively. With a brachytherapy dose of 6 Gy, the mean EQD2 doses received by the EI, II, and OB groups were 1.08, 1.49 , and $1.77 \mathrm{~Gy}$, respectively [8]. In our study, using volume based prescription to a dose of 6.5 Gy per fraction, the median EQD2 ranged between 3.92-5 Gy for point B, 1.78-2.16 Gy for the ELN groups, 2.88-3.56 Gy for the ILN groups and 4-5.2 Gy for the OBT groups across the three arms.

Lee et al. have reported in their study on the correlation coefficients between point $B$ and the dosimetric parameters of the pelvic lymph nodes. They found that D2cc to the obturator is not significantly different from the point $B$ dose. They concluded point $B$ is a poor surrogate for the pelvic lymph nodes (PLNs) [10]. Similarly, in our study we observed the point $B$ dose was not significantly different from dose to the OBT nodes across all three arms.

Lee et al. in their study showed that the total EQD2 among the lymph node groups varies from $4.1 \%$ to $9.5 \%$ of the prescribed dose and the OBT group was receiving more dose compared to other groups [11]. In our study, we observed that the total median bilateral EQD2 of BT varied from 7\% to $20 \%$ of the prescribed dose across all arms using volume based prescription.

Matsukawa et al. correlated dose to point B with dose to the pelvic lymph nodes using two applicators used in ICBT. They found that the dose delivered to point B and the PLN groups were not significantly different between TO and tandem and cylinder [12]. Similarly, we observed there was no difference in the median EQD2 to the pelvic lymph nodes across the three applicators.

There is a wide heterogeneity in EBRT boost dose to the enlarged nodes, so there is a need to determine the total lymph node dose, which includes EBRT and brachytherapy dose, to obtain complete tumour response in the nodal regions. Ramlov et al. in their study analysed 139 patients from the EMBRACE data. Median nodal boost dose was 62 Gy EQD2 (53-69 Gy EQD2). No patients had persistent nodal disease, but six patients recurred in a boosted node. Their study did not indicate any improved lymph node control with higher boost doses and concluded that there was no benefit of delivering a total lymph node dose beyond $60 \mathrm{~Gy}$ EQD2 [13]. A study by Ariga et al. tried to determine EBRT boost prescribing a median dose of 56 Gy to metastatic nodes. They found that five of 57 patients had recurred in boosted nodes [14]. Similar study by Grigsby et al. analysed different boost doses according to nodal size. A median dose of 67.2 Gy was delivered. Only five of 132 patients recurred within a boosted node [15]. Neither of the studies found a significant relation between nodal size and failure. From the above studies, we can 
infer that nodal recurrence post radiation therapy does not depend on nodal size at presentation and there is no benefit of boosting the nodal regions beyond $60 \mathrm{~Gy}$.

In our study, the total median EQD2 to the lymph nodes are arm A-52.71 Gy, arm B-53.03 Gy and arm C-53.88-62.73 Gy. This could help in deciding the EBRT boost dose as there is no difference in total EQD2 using different applicators.

The strength of our study is using volume based prescription; we documented dose to the nodal regions for three different applicators of $\mathrm{TR}$, TO and $\mathrm{TO}+\mathrm{FH}$. The limitation of our study is that it is a dosimetric analysis in which the number of patients is quite low and further clinical follow up is required. Also, there is a need for standardised guidelines for dose prescription to gross pelvic lymph nodes.

\section{Conclusion}

Our study determined the median EQD2 to the pelvic lymph nodes using volume based prescription across three types of applicators in brachytherapy. Although there is no significant difference among the applicators, there is an appreciable contribution from brachytherapy to the pelvic nodal regions. This could serve as reference to decide on the EBRT boost dose while treating patients with enlarged pelvic lymph nodes.

\section{Conflict of interest}

None declared.

\section{Funding}

None declared.

\section{References}

1. Bhatla N, Berek JS, Cuello Fredes M, et al. Cancer of the cervix uteri. Int J Gynaecol Obstet. 2018; 143 Suppl 2(1): 22-36, doi: 10.1002/ijgo.12611, indexed in Pubmed: 30306584.

2. Sakuragi N. Up-to-date management of lymph node metastasis and the role of tailored lymphadenectomy in cervical cancer. Int J Clin Oncol. 2007; 12(3): 165-175, doi: 10.1007/s10147-007-0661-2, indexed in Pubmed: 17566838.

3. Wujanto C, Choo BAi, Tan D, et al. Does external beam radiation boost to pelvic lymph nodes improve outcomes in patients with locally advanced cervical cancer? BMC Cancer. 2019; 19(1): 385, doi: 10.1186/s12885-019-5594-4, indexed in Pubmed: 31023261.
4. Oike T, Ohno T, Noda SE, et al. Can combined intracavitary/interstitial approach be an alternative to interstitial brachytherapy with the Martinez Universal Perineal Interstitial Template (MUPIT) in computed tomographyguided adaptive brachytherapy for bulky and/or irregularly shaped gynecological tumors? Radiat Oncol. 2014; 9: 222, doi: 10.1186/s13014-014-0222-6, indexed in Pubmed: 25319341.

5. Pötter R, Georg P, Dimopoulos J, et al. Clinical outcome of protocol based image (MRI) guided adaptive brachytherapy combined with 3D conformal radiotherapy with or without chemotherapy in patients with locally advanced cervical cancer. Radiotherapy and Oncology. 2011;100(1): 116-123, doi: 10.1016/j.radonc.2011.07.012, indexed in Pubmed: 21821305.

6. Rivard MJ, Coursey BM, DeWerd LA, et al. Update of AAPM Task Group No. 43 Report: A revised AAPM protocol for brachytherapy dose calculations. Med Phys. 2004; 31(3): 633-674, doi: 10.1118/1.1646040, indexed in Pubmed: 15070264.

7. Viswanathan AN, Dimopoulos J, Kirisits C, et al. Computed tomography versus magnetic resonance imaging-based contouring in cervical cancer brachytherapy: results of a prospective trial and preliminary guidelines for standardized contours. Int J Radiat Oncol Biol Phys. 2007; 68(2): 491-498, doi: 10.1016/j.ijrobp.2006.12.021, indexed in Pubmed: 17331668.

8. Chua GW, Foo YW, Tay GH, et al. Assessing dose contribution to pelvic lymph nodes in intracavitary brachytherapy for cervical cancer. J Contemp Brachytherapy. 2017; 9(4): 345-353, doi: 10.5114/jcb.2017.69237, indexed in Pubmed: 28951754.

9. Hackett TE, Olt G, Sorosky Jl, et al. Surgical predictors of para-aortic metastases in early-stage cervical carcinoma. Gynecol Oncol. 1995; 59(1): 15-19, doi: 10.1006/ gyno.1995.1261, indexed in Pubmed: 7557602.International Commission on Radiation Units and Measurements Report. Prescribing, recording, and reporting brachytherapy for cancer of the cervix. ICRU report, Bedhesda 2013 2013: Bedhesda.

10. Lee LJ, Sadow CA, Russell A, et al. Correlation of point $B$ and lymph node dose in 3D-planned high-dose-rate cervical cancer brachytherapy. Int J Radiat Oncol Biol Phys. 2009; 75(3): 803-809, doi: 10.1016/j.ijrobp.2008.11.052, indexed in Pubmed: 19286328.

11. Lee Y, Rash DL, Stern RL, et al. Variation in High-Dose-Rate Brachytherapy Dose Contribution Among Pelvic Lymph Node Groups in Locally Advanced Cervical Cancer. Int J Radiat Oncol Biol Phys. 2014; 90(1): S478, doi: 10.1016/j. ijrobp.2014.05.1482.

12. Matsukawa H, Sasaki T, Hirayama R, et al. Assessment of the anatomical position of point $B$ and the relationship between point $B$ dose and the dose delivered to pelvic lymph nodes in CT-based high-dose-rate brachytherapy for uterine cervical cancer. J Contemp Brachytherapy. 2019; 11(2): 137-145, doi: 10.5114/jcb.2019.84419, indexed in Pubmed: 31139222.

13. Ramlov A, Kroon PS, Jürgenliemk-Schulz IM, et al. Impact of radiation dose and standardized uptake value of (18)FDG PET on nodal control in locally advanced cervical cancer. Acta Oncol. 2015; 54(9): 1567-1573, doi: 10.3109/0284186X.2015.1061693, indexed in Pubmed: 26271799 . 
14. Ariga T, Toita T, Kasuya G, et al. External beam boost irradiation for clinically positive pelvic nodes in patients with uterine cervical cancer. J Radiat Res. 2013; 54(4): 690-696, doi: 10.1093/jrr/rrs138, indexed in Pubmed: 23365264.
15. Grigsby PW, Singh AK, Siegel BA, et al. Lymph node control in cervical cancer. Int J Radiat Oncol Biol Phys. 2004; 59(3): 706-712, doi: 10.1016/j.ijrobp.2003.12.038, indexed in Pubmed: 15183474. 